\title{
FIELD TEST RESULTS OF A ROAD DEPARTURE CRASH WARNING SYSTEM: DRIVER UTILIZATION AND SAFETY IMPLICATIONS
}

David J. LeBlanc, James Sayer, Christopher Winkler, Scott Bogard, Joel Devonshire University of Michigan Transportation Research Institute

Ann Arbor, Michigan, USA

E-mail: leblanc@umich.edu

\begin{abstract}
Summary: The Road Departure Crash Warning System Field Operational Test (RDCW FOT) was conducted to assess the safety impacts, driver acceptance levels, and the maturity of road departure crash warning systems as installed on a light vehicle platform. This paper presents the experimental design, performance of the road departure system in naturalistic use, and analyses of safety impacts of the technology using surrogate measures. Use of the system led to a $50 \%$ reduction in the observed rate of events in which the equipped vehicle came within $0.1 \mathrm{~m}$ of a lane edge in steady-state lane-keeping situations. Lane changes performed without the use of a turn signal were reduced by $43 \%$ on freeways and ramps and $24 \%$ on surface roads. Levels of lateral acceleration in curves was not significantly different, except on ramps, where a significant change in the $90^{\text {th }}$ percentile values of lateral acceleration were observed for a within-subject comparison. There were no observed effects of risk homeostasis and no evidence of significant negative unintended consequences.
\end{abstract}

\section{INTRODUCTION}

Single-vehicle road departures account for approximately one in five police-reported crashes in the U.S., but are responsible for approximately $40 \%$ of the highway fatalities (Emery et al., 2005). Two types of road departure events account for about half of the police-reported crashes: drivers inadvertently allowing their vehicle to drift off the road edge, and drivers entering curves too fast. These events may be reduced or mitigated using an integration of two crash warning technologies that are available or in development in the passenger car market: lateral drift warning (LDW) and curve-speed warning (CSW). A field operational test was conducted to assess the potential safety impacts, driver acceptance levels, and system maturity of an integrated LDW and CSW set. This test was conducted by the University of Michigan Transportation Institute (UMTRI) within its partnership with Visteon Corporation and AssistWare Technologies (now part of Cognex Corporation), within a cooperative agreement with the U.S. Department of Transportation.

This paper presents the experimental design, performance of the road departure system in naturalistic use, and analyses of safety impacts of the technology. A companion paper at this conference addresses the driver acceptance findings (Sayer et al., 2007). The field operational test data was also analyzed by an independent evaluation team at the Volpe National Transportation Systems Center to estimate the potential harm reduction of the system. A comprehensive report on these and other topics is also available (LeBlanc et al., 2006). 


\section{METHODOLOGY}

The RDCW FOT was conducted using a fleet of eleven passenger vehicles equipped with technologies that provided the LDW and CSW functions. The technology was developed and integrated onboard a vehicle fleet by Visteon and AssistWare/Cognex. Each vehicle was equipped with a data acquisition system to capture the driver's experiences using hundreds of data signals, two video streams, and audio. The vehicle platform was a 2003 Nissan Altima 3.5SE vehicle. (Although Nissan provided engineering information to facilitate system integration, Nissan was not involved in the project and there is no relationship between the tested systems and Nissan's systems.)

A set of drivers were recruited from the general driving population of southeast Michigan with gender split evenly and age cells of equal size containing drivers in their $20 \mathrm{~s}$, 40s, and 60s. Each test participant was trained on the RDCW system, asked to complete several pre-drive subjective instruments, given a demonstration drive, and then released for four weeks to use the test vehicle as their own. The first week of their driving was a baseline period during which driver alerts were not presented, but were captured with onboard data collection. The second, third, and fourth weeks of driving were with the RDCW system issuing driver alerts. Upon completion of the four weeks of driving, the participants returned the vehicles and completed a set of subjective instruments including questionnaires, debriefing interviews, and a joint review with a researcher using video captured during their driving experience. Some drivers returned later to participate in one of the four, two-hour focus groups. The 78 drivers used in these analyses had a travel distance exceeding 83,000 miles.

\section{SYSTEM DESCRIPTION}

The LDW system provided crash alerts intended to help drivers avoid or reduce the severity of crashes due to events of inadvertent drifting off the roadway, especially those events that are associated with driver drowsiness and distraction. The purpose of the LDW alerts was to promote the driver's awareness of their lane-keeping situation so that necessary corrections could be performed. The LDW system in this field test was based on vision-based tracking of lane markers such as painted markers or other continuous or semi-continuous visual features associated with the lane edge. The LDW system also incorporated forward- and side-looking radars to modulate alert thresholds to improve safety benefits while reducing nuisance alerts. This modulation allowed drivers to deviate further from the lane center when there were no perceived crash threats near or just beyond the lane edge. Conversely, when a crash threat such as a guardrail, a parked car ahead, or a same-direction, adjacent-lane vehicle was perceived, the thresholds were adjusted so that alerts were provided with less drift. A geo-located history of roadside objects was built onboard during each driver's experience to provide additional information. Ongoing or recent use of the turn signal or the brake pedal suppressed LDW alerts.

The CSW system issued crash alerts to avoid or reduce severity of curve-overspeed crashes; the CSW alerts were intended to prompt the driver to assess the situation and consider braking in order to reduce speed before entering the curve. This system used GPS, an onboard digital map set, and consideration of recent driver actions and lane information. The CSW system would use map-matching and anticipated road geometries to predict a most-likely path, estimate curvatures 
on that path, and provide alerts if the braking required to reduce the lateral acceleration to a given level exceeded a threshold.

\section{DRIVER CONTROLS AND DISPLAYS}

The driver could not turn off the crash warning function. The driver was able to adjust the timing of the LDW and CSW separately using two separate rocker switches located below and to the left of the steering wheel, with each warning function having five positions.

The alert displays are summarized in Table 1. For LDW, only one set of alert displays is provided for a given lateral drift event. For motion across dashed lines without a nearby adjacent-lane vehicle, a haptic "rumble strip" cue is given using motors in the seat pan. As with all alerts, a visual indicator was provided in a head-down location. For motion across a solid lane marker, or for motion across a dash marker with a nearby vehicle, an auditory tone is used. The CSW alerts include an imminent level alert (auditory voice message), and sometimes an earlier, cautionary-level alert (haptic). Subjective feedback to these designs is described in Sayer et al., 2007.

Table 1. Crash alert display modalities

\begin{tabular}{|l|l|l|}
\hline Event & Audible cue & Haptic cue \\
\hline $\begin{array}{l}\text { LDW cautionary: } \\
\text { across dashed marker, no adjacent vehicle }\end{array}$ & Pulsed directional, seat pan \\
\hline $\begin{array}{l}\text { LDW imminent: } \\
\text { across dashed marker, adjacent vehicle, OR } \\
\text { across solid marker }\end{array}$ & $\begin{array}{l}\text { Pulsed tone, } \\
\text { directional }\end{array}$ & \\
\hline CSW cautionary level (less braking required) & & Steady, seat pan \\
\hline CSW imminent level (more braking required) & Voice & \\
\hline
\end{tabular}

\section{DRIVER UTILIZATION}

The most commonly selected value of the alert timing selections was the middle of five values of each of the two controls that adjusted the relative timing of the crash alerts (one control each for LDW and CSW). Figure 1 shows the percentage of travel time associated with the different sensitivity levels. Furthermore, the adjustment of these controls was infrequent after the first week of use. Finally, $70 \%$ of the drivers used the same value of sensitivity for both the LDW and CSW systems, even though they address different driving behaviors. This may be caused in part by the difficulty of receiving feedback on the choice of CSW setting, since a driver cannot provoke a CSW alert without a relatively sharp curve. Therefore, a driver has less opportunity to adjust the setting and test (or experience) the results

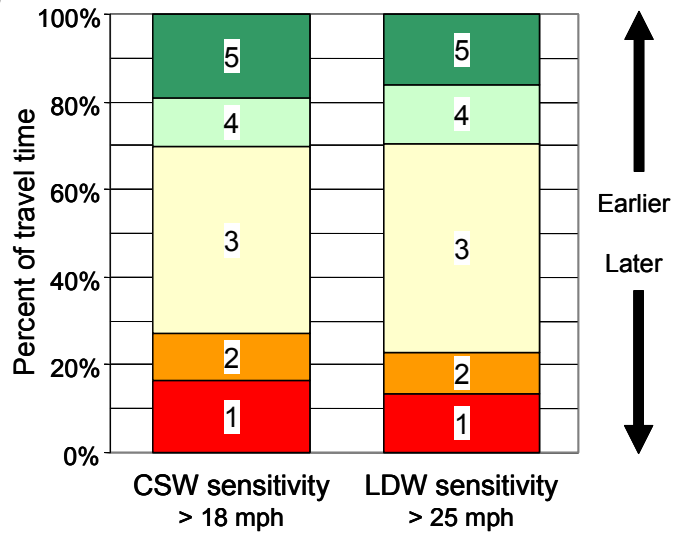

Figure 1. Driver settings of sensitivity of that adjustment than with other systems. 


\section{CRASH ALERT EXPERIENCE}

Drivers experienced an average of 74 LDW alerts (28 cautionary and 46 imminent), and 46 CSW alerts ( 32 cautionary and 14 imminent). The median numbers were $60 \mathrm{LDW}$ alerts (15 cautionary and 40 imminent) and $38 \mathrm{CSW}$ alerts (28 cautionary and 12 imminent). There was considerable variation between drivers, with more than two orders of magnitude between drivers with the fewest and the greatest number of alerts. When normalizing by travel distance, the average rates were 10.6 and 6.1 alerts per 100 miles $(161 \mathrm{~km})$, respectively, for LDW and CSW. Given typical travel speeds, this translates to very roughly three LDW and two CSW alert events per hour of travel, as an average rate over the field test.

The qualitative nature of the crash alerts is significant. A set of $884 \mathrm{CSW}$ alerts and $854 \mathrm{LDW}$ alerts were sampled and reviewed in detail in order to assign characteristics of the driving event. Half of the sampled alerts within each warning functionality were cautionary and the other half were imminent alerts. Table 2 shows a breakdown of the fraction of LDW and CSW alerts that were associated with different driving scenarios and driver responses. For LDW, note that 17\% of the sampled alerts were judged to be false alerts. Furthermore, the table allows one to compute that for the sampled LDW alerts (excluding false alerts), the driver "corrected" or moved the vehicle back toward the center of the original travel lane only $29 \%$ of the time. Sayer et al. (2007) reports on driver ratings of alert utility using this scenario classification. Table 2 also shows that $43 \%$ of the CSW alerts reviewed were associated with system technical issues. These cases fell into one of three categories: predicting that drivers would take a branch with a sharp curve when in fact they did not do so; technical problems (alerts that were caused by a unit rebooting onboard); issues associated with the use of navigation maps for this application; and events that were not classified due to difficulties in relating the alert event to an upcoming curve. Thus, it is estimated that approximately one in two CSW alerts occurred when the driver did not eventually traverse the curve that triggered the alert.

Table 2. Scenarios in which LDW and CSW alerts occurred

\begin{tabular}{|l|l|r|}
\hline \multirow{4}{*}{$\begin{array}{l}\text { LDW } \\
\text { alert } \\
\text { events }\end{array}$} & False alert (lane tracking or threat positioning) & $\mathbf{1 7 \%}$ \\
\cline { 2 - 3 } & Alerted while drifting in lane - didn't correct after alert & $\mathbf{1 4 \%}$ \\
\cline { 2 - 3 } & Alerted while drifting in lane - corrected after alert & $\mathbf{1 1 \%}$ \\
\cline { 2 - 3 } & Alert during un-signaled lane change & $\mathbf{3 3 \%}$ \\
\cline { 2 - 3 } & Alert while drifting out of lane - didn't correct after alert & $\mathbf{1 2 \%}$ \\
\cline { 2 - 3 } & Alert while drifting out of lane - corrected after alert & $\mathbf{1 3 \%}$ \\
\cline { 2 - 3 } & All sampled LDW alert events & $\mathbf{1 0 0 \%}$ \\
\hline \multirow{4}{*}{$\begin{array}{l}\text { CSW } \\
\text { alert } \\
\text { events }\end{array}$} & Curve triggering alert was traversed after alert \\
\cline { 2 - 3 } & Curve triggering alert was not traversed after alert & $\mathbf{2 5 \%}$ \\
\cline { 2 - 3 } & System issues or unclassified alerts & $\mathbf{4 3 \%}$ \\
\cline { 2 - 3 } & All sampled CSW alert events & $\mathbf{1 0 0 \%}$ \\
\hline
\end{tabular}




\section{POTENTIAL SAFETY IMPACTS}

Regarding safety implications of the system, there were four major findings. The first is that the use of turn signals increased, especially for those drivers who did not use turn signals often during the baseline period. This was considered to be the results of two factors. First, the LDW alerts were completely suppressed when a turn signal was used. Most drivers were sent to use the turn signal more when the LDW was active. This may be caused by the driver's desire to avoid receiving an alert. In addition, several drivers reported that the LDW made them aware that their lane-keeping and attention performances were poor, so the use of turn signals may also be caused by this enhanced awareness of lane management. Figure 2 shows the percentage of lane changes in which the driver did not use the turn signal by week, where the first week was the baseline period (without driver alerts). The fraction of un-signaled lane changes was reduced by $43 \%$ on freeways and $24 \%$ on surface roads.

The second finding was the result of studying steady-state lane-keeping performance with and without the driver alerts. A set of data corresponding to 183 hours of driving was parsed from the data by imposing a set of conditions on the data, including: travel speed of at least $25 \mathrm{mph}$ (11.4 $\mathrm{m} / \mathrm{sec}$ ), a known road type, lane tracking successful on both lane edges, no braking activity, and a lane position with at least one tire in the original travel lane. Furthermore, each episode was required to be at least $36 \mathrm{sec}$ in duration and the first and last eight seconds of data was discarded to remove the confound of lane changes that might bound the episode. Figure 3 shows that the rate of events in which the vehicle edge came within $0.1 \mathrm{~m}$ of the lane edge was reduced by $50 \%$, when considering both sides. Similar statistically significant findings emerged when different metrics were considered, such as the standard deviation (reduction by $11 \%, \mathrm{p}<0.003$ ).

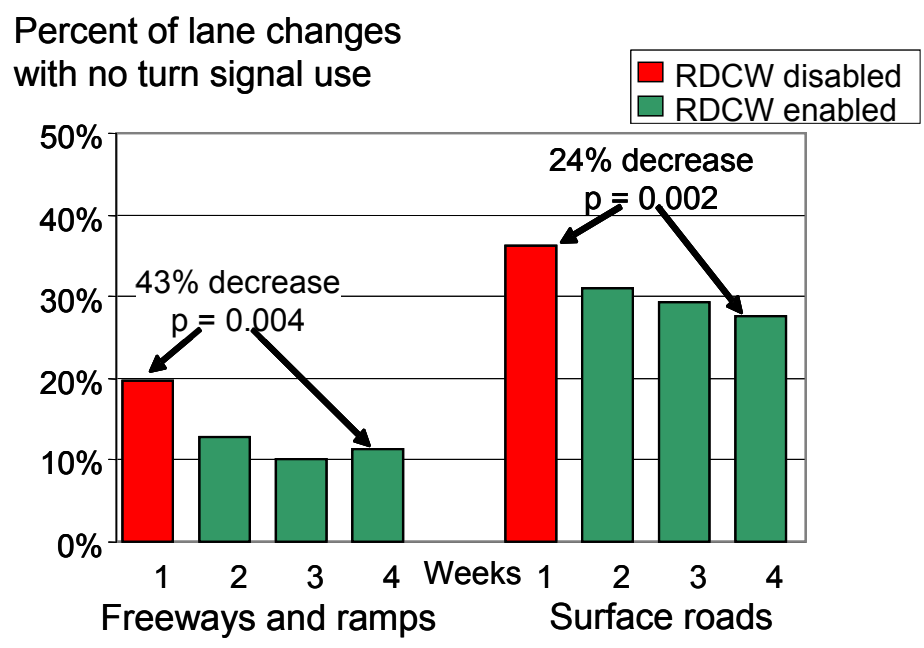

Figure 2. Increase in use of turn signals during lane changes 


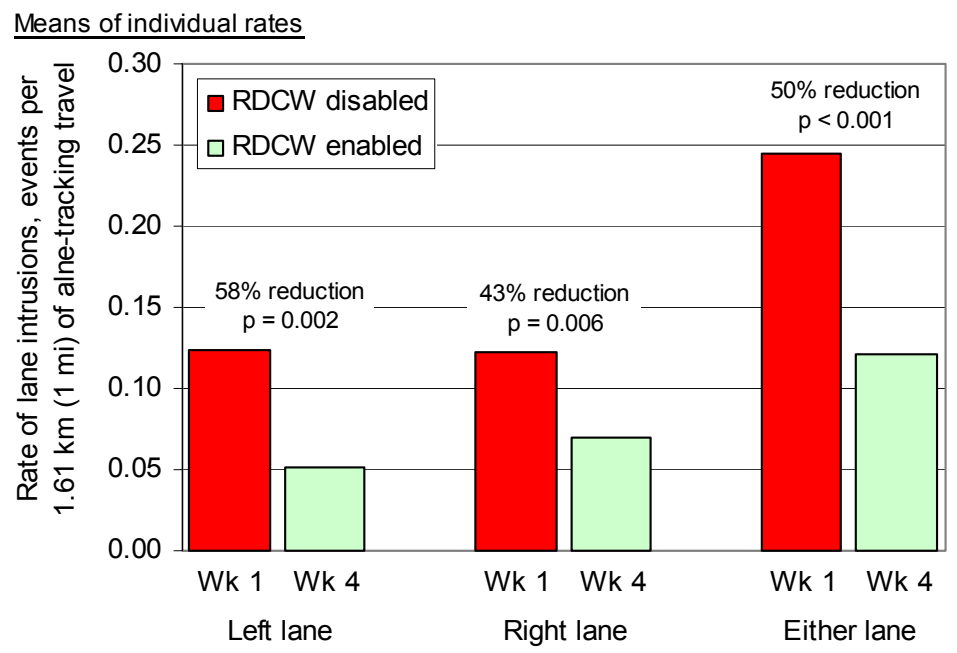

\section{Figure 3. Reduction in the rate of events in which vehicle edge comes within $0.1 \mathrm{~m}$ of lane edge}

The third finding was that there was insufficient evidence to confirm the hypothesis that drivers traveled through curves at different speeds when the CSW system was available. The analysis used the lateral acceleration parallel to the road surface as the most important metric. A set of statistical tests were conducted to look for main or interacting effects based on multiple metrics associated with lateral acceleration. To do this, consideration of multiple factors was done within a multivariable framework, including road type (ramp vs. freeways vs. surface roads), whether the wipers were on, driver age, direction of the curve (left vs. right), and daytime vs. nighttime. The results are summarized in Table 3, showing that the only case in which a statistically significant effect was observed in the presence of the RDCW crash alerts was curve-taking on

Table 3. Lateral acceleration in curves: trends with significance of $\mathrm{p}<0.05$

\begin{tabular}{|l|l|}
\hline \multicolumn{1}{|c|}{ Factor } & \multicolumn{1}{c|}{ Higher lateral accelerations for... } \\
\hline Road type & Ramps $>$ Local \& surface $>$ Freeway \\
\hline Wiper state & Wipers off ("dry") > Wipers on ("wet") \\
\hline Driver age & Younger $>$ Middle age $>$ Older \\
\hline Direction of curve & Curving right $>$ Curving Left \\
\hline Ambient lighting & Daytime $>$ Nighttime \\
\hline RDCW-ramps & Before RDCW $>$ After RDCW \\
\hline RDCW / other cases & No significant findings \\
\hline
\end{tabular}
ramps, and this was only when studying the $90^{\text {th }}$ percentile of lateral acceleration in ramps with and without RDCW cues.

This lack of support for the hypothesis that curve-taking performance would be altered by the system does not necessarily mean that CSW does not affect curve-taking performance. The experimental design was noted to be less than ideal for studying curve-speed warning, since several drivers commented that they would find the system most useful in poor weather or in unknown environments. Furthermore, the amount of data collected at lateral accelerations above the nominal threshold level that CSW attempted was very small. Figure 4 shows that none of the 78 drivers had a $90^{\text {th }}$ percentile value that exceeded the CSW threshold. 


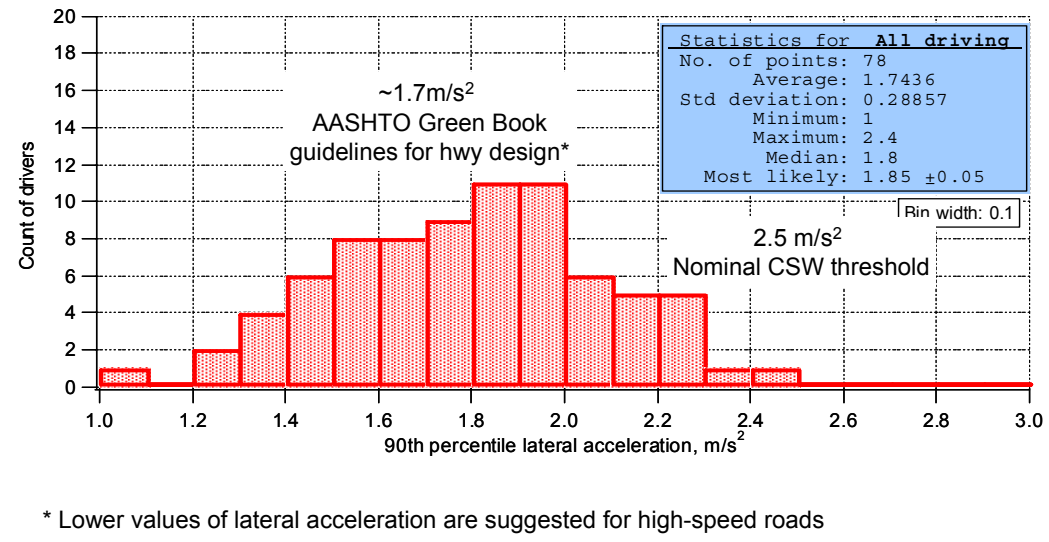

Figure 4. $90^{\text {th }}$ percentile values of lateral acceleration in curves for individual drivers

The fourth finding was that there was no clear indication of unintended consequences or of risk compensation by drivers.

\section{CONCLUSIONS}

When driving with the road departure warning system, the test participants changed lane-keeping and turn-signal behaviors that may result in safety benefits. There is inconclusive evidence about whether lateral acceleration in curves would be reduced since the testing did not include substantial amounts of unsafe travel in curves. The companion paper will address driver acceptance and willingness to purchase both systems

\section{ACKNOWLEDGMENTS}

The authors thank colleagues at Visteon (Debby Bezzina, Tim Tiernan, Faroog Ibrahim, Dinu Madau, and others) and AssistWare/Cognex Corporation (Dean Pomerleau). Nissan Corporation graciously provided engineering information to facilitate system integration into the vehicle. The work was conducted under agreement DTFH61-01-X-00053 with the U.S. DOT. Our thanks to Lloyd Emery (retired) and Ray Resendes of NHTSA and Bruce Wilson of the Volpe Center.

\section{REFERENCES}

Emery, L., Srinivasan, G., Bezzina, D., LeBlanc, D., Sayer, J., Bogard, S., and Pomerleau, D. (2005). Status report on USDOT project 'An intelligent vehicle initiative road departure crash warning field operational test.' Proc. $19^{\text {th }}$ Intl. Technical Conference on the Enhanced Safety of Vehicles, US DOT HS 809 825, Washington, DC.

LeBlanc, D., Sayer, J., Winkler, C., Bogard, S., Devonshire, J. Mefford, M., Hagan, M., Bareket, Z., Goodsell, R., and Gordon, T. Road departure crash warning system (RDCW) field operational test final report. UMTRI Technical Report 2006-9-1.

Sayer, J., LeBlanc, D., Mefford. M.L., and Devonshire, J. (2007). Field test results of a road departure crash warning system: utility and willingness to purchase. Proc. 4th Int. Driving Symp. on Human Factors in Driver Assessment, Training, \& Vehicle Design. Stevenson, WA. 